\title{
Models for the Generation and Interpretation of Gradients
}

\author{
Hans Meinhardt \\ Max-Planck-Institut für Entwicklungsbiologie, Spemannstr. 35, D- 72076 Tübingen \\ Correspondence: hans.meinhardt@tuebingen.mpg.de
}

Source regions for morphogen gradients_-organizing regions_can be generated if a local self-enhancing reaction is coupled with a long-ranging reaction that acts antagonistically. Resulting gradients can be translated into patterns of stable gene activities using genes whose products have a positive feedback on the activation on themselves. If several autoregulatory genes compete with each other for activity, cells make an unequivocal choice. Although the signal consists of a smoothly graded distribution, the all-or-nothing response of the cells leads to regions of differently determined cells that are delimited by sharp borders. In some systems, it is not the absolute but the relative level of a gradient that matters. The sequence of head, tentacles, and foot formation in hydra is controlled by a head activation gradient and is an example of this widely used but conceptually rather neglected mode. For subpatterns such as legs and wings, different "compartments" cooperate to produce new signaling substances. Here, morphogen production is restricted to the common borders or where they intersect. The model accounts for the formation of substructures in pairs at the correct positions within the embryo and for the correct orientation and handedness with respect to the main body axes.

M any observations in developmental biology can be accounted for by the assumption that graded distributions of substances control cell differentiation in a concentration-dependent manner. Earlier objections against this concept came from two sides. First, the assumption of gradients shifts only the problem as long as no explanation is given of how the gradients are generated in the first place. Second, cells must be extremely sensitive in measuring the absolute concentrations. Only minute concentration differences are expected to exist between adjacent cells. Nevertheless, these must be sufficient to accomplish a reliable and unequivocal selection between alternative pathways.
Long before the molecular approach became feasible, we proposed models showing that these features can be realized by molecularly reasonable interactions. Meanwhile, these models found strong support by molecular and genetic observations.

\section{ORGANIZER FORMATION BY LOCAL SELF-ENHANCEMENT AND LONG-RANGE INHIBITION}

For the generation of gradients within an initially more or less homogeneous assembly of cells, local source regions have to be generated. Although maternally supplied asymmetries often play a role, clear evidence exists that

Editors: James Briscoe, Peter Lawrence, and Jean-Paul Vincent

Additional Perspectives on Generation and Interpretation of Morphogen Gradients available at www.cshperspectives.org

Copyright (C) 2009 Cold Spring Harbor Laboratory Press; all rights reserved; doi: 10.1101/cshperspect.a001362

Cite this article as Cold Spring Harb Perspect Biol 2009;1:a001362 
pattern formation can start from a more or less uniform initial situation. For instance, any cell of an eight-cell mouse embryo can give rise to a complete embryo, showing that at this stage each cell retains the full potential to form the complete organism. After initial proliferation of such an isolated cell, it is to be expected that all the daughter cells are more or less identical, requiring a genuine pattern-forming process for axes formation. After fragmentation of an early chick embryo, each fragment can lead to a complete embryo, even if the fragment does not contain the putative organizing region (Lutz 1949). While in a fragment that contains the organizer. The embryo forms with a predictable orientation. The orientation of the embryos in the remaining fragments is highly variable, indicating again that minor differences or even random fluctuations are decisive. In the freshwater polyp hydra, perfectly normal and viable animals can develop even after dissociation into individual cells and reaggregation (Gierer et al. 1972). Likewise, in Xenopus, coculture of dissociated animal and vegetal cells leads not only to mesoderm induction but also to the establishment of new organizing regions as indicated by the formation of notochord and neural tube (Nieuwkoop 1992). Cell dissociation certainly wipes out any maternally imposed asymmetries. Moreover, in many systems, the organizer can be removed and, nevertheless, development proceeds normally (Harland and Gerhart 1997; Yuan and Schoenwolf 1998; Saùde et al. 2004). These observations show that at least in these cases development is not fixed by mosaic-like pre-existing distributions of determinants and that sources of morphogens are regulated in a highly dynamic manner.

We have shown that primary pattern formation is possible if, and only if, a locally restricted self-enhancing reaction is coupled with an antagonistic reaction that acts on a longer range (Gierer and Meinhardt 1972; Gierer 1977a,b; Meinhardt 1982, 2008). One can show that pattern formation in the inorganic world, such as the formation of sand dunes, clouds, all forms of erosion, etc., is based on the same principle. In a pioneering paper, Alan Turing (1952) has shown that the interaction of two substances with different diffusion rates can lead to pattern formation. He coined the term "morphogen." However, in his paper the crucial requirement-local self-enhancement and long-range inhibition-is not incorporated. Knowing the generative principle allowed us to derive a general criterion defining which interactions lead to stable patterns and which do not. It allowed us further to include nonlinear interactions, which are indispensable to derive molecularly feasible schemes.

A prototype of such a pattern-forming reaction consists of a short-ranging substance we called the activator, which promotes-directly or indirectly-its own production. It also regulates the synthesis of its rapidly diffusing antagonist, the inhibitor. The latter slows down the autocatalytic activator production (Fig. 1) or catalyzes the activator decay. A homogeneous distribution is unstable. For example, a small local elevation of the activator will increase further because of autocatalysis, although a surplus of the inhibitor is also produced at the same position. This additional inhibitor diffuses rapidly into the surroundings, regulating down the activator production there, while at the local elevation, the activator concentration increases further. As shown in Figure 1, a new patterned steady state is reached when the local high activator concentration is in a dynamic equilibrium with the surrounding cloud of the inhibitor. Both the more localized activator and the more smoothly distributed inhibitor can be used as a morphogenetic signal.

Pattern formation requires a certain field size so that the different diffusion rates can come into play. If this critical size is attained in a growing embryo, a high concentration emerges at one side and a low concentration at the opposite side (Fig. 1). The generation of such a pattern is a most important step. Although the genetic information is the same in all the cells, different genetic information can be activated in a position-dependent manner. Thus, our model provides a solution for one of the most puzzling features of development: How can distinct spatial structures emerge in a reproducible way even when starting from a more or less structureless initial situation? 
Models for the Generation and Interpretation of Gradients
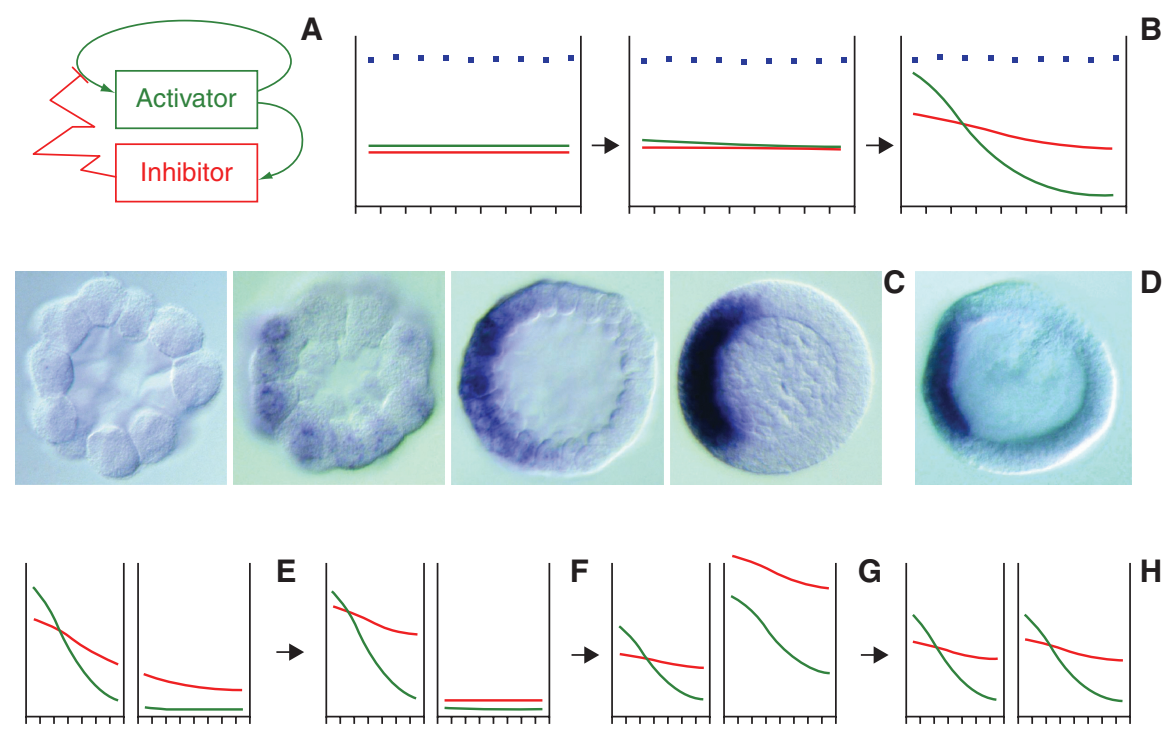

Figure 1. Pattern formation by an activator-inhibitor interaction. (A) Reaction scheme: The activator catalyses its own production. The production of its rapidly spreading antagonist, the inhibitor, is also under activator control (Gierer and Meinhardt 1972; Meinhardt 1982, 2008). (B) In such a reaction, the homogeneous distribution of both substances is unstable. Small random fluctuations in the ability to produce these substances (blue squares) are sufficient to initiate pattern formation. A high concentration appears at a marginal position. Thus, although the genetic information is the same in all cells, such a system is able to generate a reproducible polar pattern, appropriate to accomplish space-dependent cell differentiation (see Fig. 3). (C) A biological example: the emerging Nodal gradient in the sea urchin, responsible for the formation of the oral field. (D) Antivin (or Lefty2, left) acts as inhibitor (Duboc et al. 2004). As predicted, it is produced at the same position as the activator. $(E-H)$ Regeneration. After fragmentation, in the nonactivated fragment, the remnant inhibitor disappears $(F)$ until a new activation is triggered $(G)$. The graded profiles are restored $(H)$ as long as the remaining fragment is still large enough. Because the inhibitor can escape only into a smaller nonactivated region, the activations are somewhat reduced (Fig. C kindly provided by Dr. Thierry Lepage).

At the time the theory was proposed (Gierer and Meinhardt 1972), our activator-inhibitor systems were completely hypothetical. Since then, several systems have been found that correspond to this scheme. A recently found example is the formation of the nuclear localization of Dorsal in Tribolium (Nunes da Fonseca et al. 2008). It depends on a selfenhancing process involving Toll, which is antagonized by cactus. A further example is the Nodal/Lefty2-system. Nodal is a secreted factor that has a positive feedback on its own production. Lefty 2 is under the same control as Nodal and acts as an antagonist. In contrast to Nodal, Lefty 2 cannot dimerize and blocks in this way the Nodal receptor. This system is involved in the formation of the oral field in the sea urchin (Fig. 1C) (Duboc et al. 2004) and in the left/right patterning of vertebrates
(Chen and Schier 2002; Nakamura et al. 2006; for modeling, see Meinhardt 2001).

The model accounts not only for the generation but also for regeneration of a pattern (Fig. 1E-H). By the removal of an area of high activator concentration, the area of inhibitor production is also removed. After the decay of the remnant inhibitor, the formation of a new activator maximum is triggered in the remaining cells, starting from a low level activator production. The pattern becomes restored in a self-regulatory way.

\section{THE SPEMANN-TYPE ORGANIZER AS A PATTERN-FORMING REACTION}

The Spemann-type organizer is a central paradigm for pattern formation in vertebrates (see Plouhinec and De Robertis 2009). Central in 
organizer formation is the mutual inhibition of $B M P$ and Chordin. The antagonistic action of Chordin on $B M P$ leads to a $B M P$ gradient that has its low point at the organizer. This inverted $B M P$ gradient may organize the mediolateral pattern in vertebrates (Dosch et al. 1997). As mentioned, organizer formation in vertebrates has many features that indicate a self-organizing patterning process. For instance, in many systems, the organizer can be removed and, nevertheless, normal pattern formation follows, suggesting that organizer formation is based on a genuine pattern-forming process with corresponding regulatory properties.

In terms of our model, the selfenhancement required for organizer formation need not to be direct. Two components that mutually inhibit each other produce positive autoregulation: A concentration increase of one component leads to an enforced repression of the other, which, in turn, leads to a further increase of the first as if this substance would be directly autoregulating. Thus, the required autoregulation for the formation of the Spemann organizer is proposed to be realized by mutual inhibition of Chordin and BMP (Fig. 2). To obtain a balanced regional activation of Chordin on BMP, a third component is required that acts antagonistically on one of these indirectly self-enhancing reactions. A candidate is the antidorsalizing morphogenetic protein (ADMP) (Moos et al. 1995). Its properties have been frequently regarded as counterintuitive: being expressed in the organizer, but functioning by reducing organizer activity. However, as it acts over a longer range (Lele et al. 2001; Willot et al. 2002; Reversade and De Robertis 2005), it satisfies our theoretical expectations: being produced in the organizer region, and yet antagonizing a self-enhancing reaction. Figure 2 shows the generation of a pattern based on such a mechanism (for equations and further details, see Meinhardt 2008). Although the actual mechanism is even more complex (see Barkai and Shilo 2009), this simplified scheme accounts for many observations, such as broadening and shrinking of expression regions if $B M P$, Chordin, or ADMP are miss-expressed. The regeneration of the organizer after ablation is a consequence of the model (Fig. 2E).

\section{GRADIENTS FOR THE PRIMARY \\ BODY AXES IN VERTEBRATES}

There are two primary body axes, anteroposterior (AP) and dorsoventral (DV). Usually, however, only one organizer is assumed to exist-the Spemann-type organizer. How can two orthogonal positional information systems emerge under the influence of a single organizer? Is there a second organizer, which has been so far overlooked? There are good arguments that the organizer for the AP pattern is not the Spemann-organizer but the entire marginal zone (Meinhardt 2006). In the early gastrula, Wnt is produced in the marginal zone except for the organizer region (Christian and Moon 1993). Wnt provides positional information for the separation into fore- and midbrain (Kiecker and Niehrs 2001; Nordström et al. 2002; Dorsky et al. 2003). This patternforming system is evolutionarily very old. A comparison of gene expressions in hydra and the early vertebrate gastrula shows a surprising correspondence, suggesting that patterning of the vertebrate brain and heart evolved from a system that was once responsible for the patterning of the body of a hydra-like ancestor (Fig. 2B) (Meinhardt 2002). In this view, the hydra organizer and the vertebrate blastopore, i.e., marginal zone, germ ring, etc., are homologous structures that are responsible for the AP patterning.

In contrast to the hydra organizer, the vertebrate blastopore evolved into a huge ring with Spemann organizer forming a small patch on this ring. The Spemann organizer is then assumed to pattern the DV axis, but it does so indirectly by giving rise to the dorsal midline, the notochord, and floor plate - a "high line" and not a "high spot" for the DV patterning. Both organizing regions, the blastopore for the AP and the midline for the DV axis, form a near Cartesian coordinate system that allows a combinatorial patterning along both axes (Fig. 2C). The generation of a single longextended "high line" for the DV patterning is a 
Models for the Generation and Interpretation of Gradients
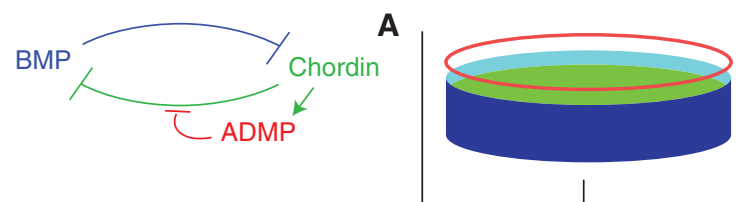

D

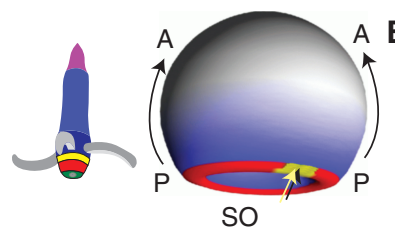

A
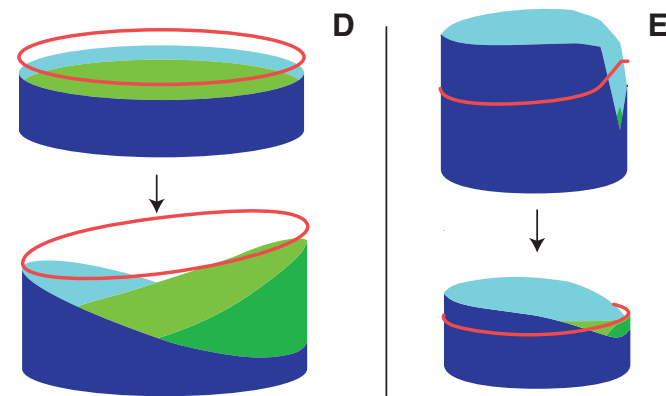

E

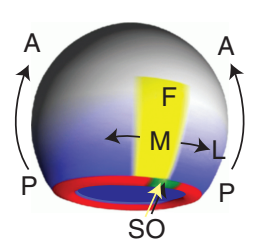

C
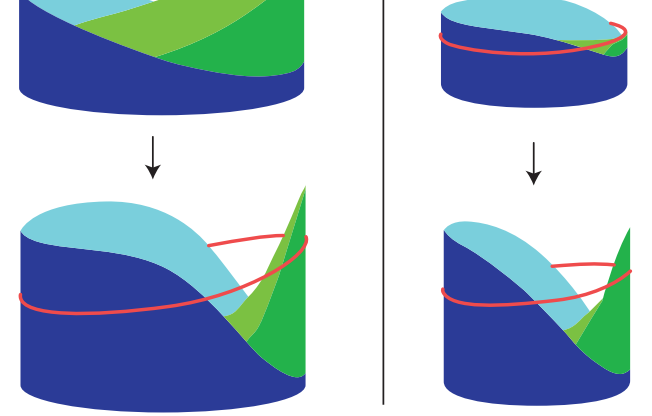

$\downarrow$

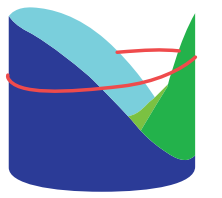

Figure 2. Model for the dorsoventral (DV) patterning in vertebrates and the geometry of axis formation. (A) Reaction scheme: The mutual inhibition of Chordin and BMP generates a system with self-enhancing properties as required for pattern formation. $A D M P$ is assumed to act as the inhibitor because it is under the same control as Chordin, and diffuses more rapidly. It blocks the self-enhancing process by undermining the $B M P$ repression exerted by Chordin. (B) Geometry: The ancestral organizer of a hydra-like ancestor became a large ring in vertebrates, e.g., the marginal zone in amphibians (red: Brachyury expression). The Wnt signal (fading blue), produced in marginal zone, accomplishes the anteroposterior (AP) specification. The Spemann-organizer (SO) can only be formed in the marginal zone. $(C)$ With mesoderm ingression, the organizer-derived mesodermal cells form the prechordal plate (yellow) that acts as line of reference for the mediolateral organization. Both organizers together set up a perfect Cartesian coordinate system: the marginal zone for the AP and the prechordal plate for the DV or mediolateral axis. (D) Simulation of pattern formation according to the scheme $(A)$. Even when starting from homogeneous distributions, a sharp Chordin peak (green) emerges. $B M P$ has a complementary distribution and is assumed to provide positional information for the DV axis. (E) After organizer removal, the organizer regenerates (for details, see Meinhardt 2006, 2008).

subtle pattern-forming process. The vertebrate solution is not the only one. In insects, for instance, a dorsal organizer exerts a repressing influence, causing the midline to appear at the opposite ventral side (Meinhardt 2004, 2008), much in contrast to vertebrates in which the organizer initiates and elongates the midline dorsally. This model provides a rational for the dorsal or ventral location of the central nervous system in vertebrates and insects, respectively.

\section{INTERPRETATION OF POSITIONAL INFORMATION: SWITCH-LIKE GENE ACTIVATIONS BY POSITIVE AUTOREGULATORY FEEDBACK}

Signals generated by diffusible molecules are necessarily transient. The communication between different parts would require more and more time in the tissue enlarging by growth. Moreover, the slope of a gradient depends on the half-life and the diffusion rate of the signaling molecule, and would not automatically adapt to a growing field size. Therefore, at early stages, the diffusiongenerated signals have to be translated into more stable states of cell determination that can be maintained independent of the inducing signals. An obvious means is a concentration(and thus space-) dependent activation of genes.

There is a formal analogy between gene activation and pattern formation. Spatial pattern formation requires an activation at a particular position and the inhibition elsewhere. The selection of a particular pathway requires the 
activation of a particular gene and the suppression of alternative genes. Thus, essential steps in development can be regarded as a sequence of patterning processes in real space coupled with pattern formation among alternative genes. This formal analogy was the rationale for predicting that cell determination requires autoregulatory gene activation (Meinhardt 1976, 1978). The long-range inhibition in spatial pattern formation corresponds to the repression of the alternative genes in gene activation.

A stable switch-like activation of a single gene can result from a nonlinear saturating autocatalytic feedback of a gene product on the activation of its own gene (Meinhardt 1976, 1978). Meanwhile, many genes with positive autoregulation are known. The gene deformed in Drosophila is an example (Regulski et al. 1991). Because of autoregulation, a short activation of the deformed gene under heat shock control is sufficient for a long-lasting activation of this gene (Kuziora and McGinnis 1988). Two Deformed molecules have to bind to each other to be able to activate the deformed gene. This nonlinearity was predicted to be crucial for the switch-like activation. The rational is easy to understand. At low concentration, the chance of finding a partner for building a dimer is low. Therefore, the normal first-order decay is dominating and the level of the gene product will remain low. The morphogen signal is assumed to have an additional activating influence on this gene activation. With increasing signal concentration, that rate of dimerization increases too. From a certain threshold level onwards, the rate of the nonlinear autoactivation becomes larger than the first-order decay rate and the gene activation switches from OFF into ON state. Because of a maximum rate in the autoregulation, the gene activation reaches a stable high level. The activation can remain in the $\mathrm{ON}$ state even if the signal is no longer available.

For organizer formation (Fig. 2) and gene activation, the required autoregulation can be realized by an inhibition of an inhibition. An example is the unambiguous specification of the border cells in Drosophila. Border cells delaminate together with the polar cells from the sheet of follicle cells, and move to the oocyte. The molecular base of this switching is now well understood. The polar cells generate a gradient that activates the JAK/STAT pathway in the surrounding follicle cells, initially causing a graded JAK/STAT activation. However, JAK/STAT activates two components that inhibit each other, APT and, at higher levels, SLOBO. APT on its own would suppress JAK/STAT everywhere. At high JAK/STAT levels, SLOBO suppresses the action of APT. The initially graded JAK/STAT activation becomes converted into a steplike activation, providing a clear decision of which cells do form border cells and which do not (StarzGaiano et al. 2008).

According to the classical view, morphogen gradients lead to an ordered activation of several genes because of a concentration-dependent response. How do cells measure the local concentration with such a precision? An analysis of ligation experiments with non-Drosophila insects suggested that cells do not measure different levels in a single step but that they compare their achieved state of determination with the strength of the external signal (Meinhardt 1978). A sequential transition from one gene activation to the next will occur as long as the signal is still high enough to accomplish the next switch. Usually, this sequential activation of genes specifying more posterior or more distal structures is essentially irreversible when completed (Fig. 3). According to this model, the cells become determined according to the highest concentration they were exposed to in their past. Therefore, a later fading of the signal because of an increasing distance between the cells and the signaling source has no effect. In this mechanism, there is no direct communication between adjacent structures. The correct neighborhood depends solely on the interpretation of the graded signal. This has the consequence that mismatches caused by transplantation at later stages might be neither detected nor repaired.

A characteristic feature of such systems is that determination can be changed only 
Models for the Generation and Interpretation of Gradients

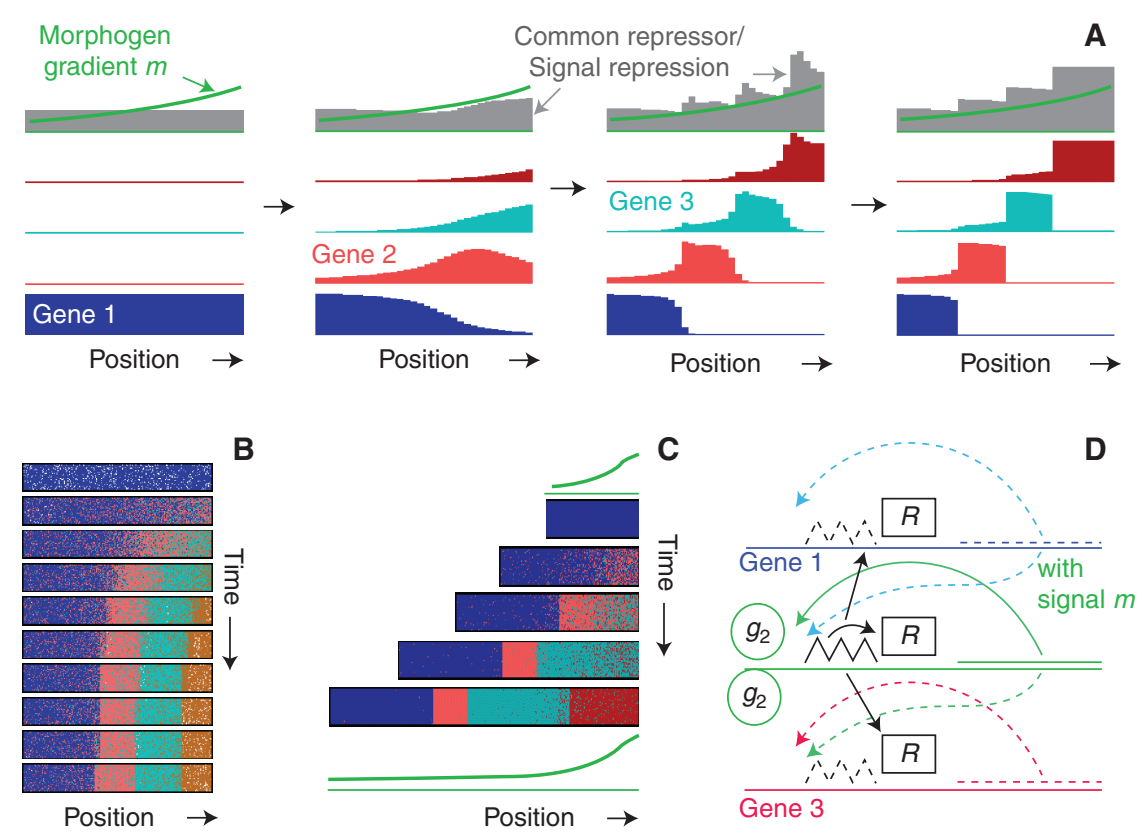

Figure 3. Space-dependent activation of several genes under the influence of a morphogenetic gradient. The genes whose gene products have a positive and nonlinear feedback on the activation of their own gene are assumed. They compete with each other for activity. (A) Starting with a default activation of the gene 1 (blue), the genes 2, 3, and 4 become successively activated. Regions with sharp borders are formed. In a given cell, only one of the alternative genes can be active. The sequential activation of genes proceeds faster in regions of high signal concentration, which leads to the apparent wavelike movement of gene activities. Activation of new genes occur only unidirectionally (distal or posterior transformation). With the activation of a further gene, the concentration of a common repressor (grey) increases. In the model, this reduces the sensitivity for the signal, causing the sequential gene switching to come to rest according to the local signal level. (B) A simulation as shown in $(A)$; the gene activities are plotted as densities of pixels, analogous to what is seen in in situ hybridizations. The slowing down of the wavelike movement of particular gene activities and the sharpening of the borders is clearly visible. $(C)$ A short-ranging gradient can control a larger region if cell proliferation is essentially restricted to the source region. Cells leaving the source region because of proliferation enter a region of lower signal strength and attain a stable determination. Evidence for such a mechanism exists for the determination of the digits in the chicken wing bud (Harfe et al. 2004; see also Bénazet and Zeller 2009). (D) Scheme: several autoregulatory genes are assumed that locally compete with each other either by a common repressor $R$ or by a direct negative cross-regulation between alternative gene products $g_{1}, g_{2} \ldots$ (Meinhardt 1978, 1982).

in a unidirectional way (distal or posterior transformation). Therefore, on transplantation from a region of high to a region of low concentration, the cells maintain their already achieved determination. In contrast, after a low-to-high transplantation, the cells change their determination according to the new level. Strong evidence for such a unidirectional promotion exists for the hindbrain (Gould et al. 1998; Grapin-Botton et al. 1998), in the commitment of CNS progenitors along the DV axis of Drosophila neuroectoderm, and for the response to activin signaling in the early amphibian gastrula (Gurdon et al. 1995). A stepwise posterior transformation was proposed for the AP specification in the anterior neural tube (Nieuwkoop 1952).

The stepwise and time-requiring "promotion" opens the possibility that gradients with relatively short ranges can be used to pattern a larger region. Required for this is that cell proliferation takes place predominantly in the source region (Fig. 3C). Cells leaving the source region are fixed in their determination 


\section{H. Meinhardt}

because they move into a region of lower morphogen concentration, whereas cells in the source region can still be further promoted. Evidence for such a mode has been found in the interpretation of the Shh gradient for the AP patterning of the vertebrate limb (Harfe et al. 2004).

The maintenance of the determined cell state by feedback of a gene on its own activity, combined with a repression of alternative genes, seems to be a widespread mechanism. It is, however, not the only one. Another mechanism is based on changes in the chromatin packaging, e.g., by DNA methylation. This leads to a different accessibility of particular genes in the chromatin-a mechanism that will be not considered here (see Ringrose and Paro 2007; Schwartz and Pirrotta 2007).

\section{IF RELATIVE LEVELS MATTER: GRADIENTS OF COMPETENCE FOR ORGANIZER FORMATION}

In most models for cell response to morphogenetic gradients, it is the absolute concentration of a graded substance that is assumed to determine the activation of a particular gene. However, as an analysis of the head-, tentacle-, and foot-formation in hydra has shown, there are also systems in which the relative levels of a graded distribution are decisive (Fig. 4).

If a hydra (or a planarian) is fragmented, regeneration occurs by maintaining the original polarity (see Adell et al. 2009; Bode 2009), independent of the original position of the fragment within the animal. The maintenance of polarity during regeneration indicates that tissue regions that were once closer to the original head have a
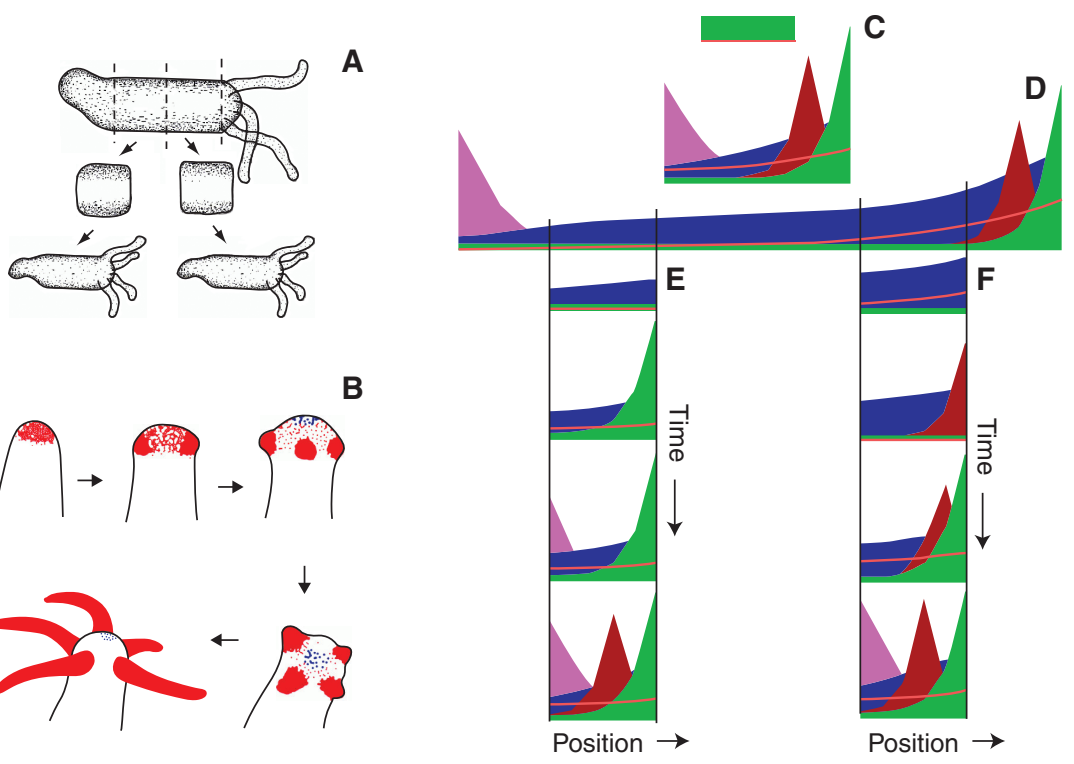

Figure 4. Patterning in hydra, a system in which relative levels of a gradient are important. ( $A$ ) Small fragments of hydra always regenerate with the original polarity. (B) During regeneration, the signal for tentacle formation appears first in the cells at the extreme tip and shift later to the appropriate position (Bode et al. 1988; signal for tentacle formation is visualized by antibodies, shown here in red). $(C),(D)$ Model: The signals for the formation of the head (green), tentacles (dark red), and foot (pink) are assumed to be generated by separate activator-inhibitor systems. These systems are positioned by source density gradient (blue) that is elevated by the head-system and down-regulated by the foot-system. The source density gradient corresponds to the head activation gradient in the hydra literature. Tentacles appear in the region of the highest source density that is not occupied by the head signal. $(E),(F)$ Small fragments regenerate all signals with the correct polarity. The head is formed at the relatively highest levels of the residual head activation gradient, the foot at the lowest. The temporary formation of the tentacle signal at the very tip as shown in $(B)$ is correctly described (F) (simulations after Meinhardt 1993; see also Bode 2009). 
better chance to win the competition to form the new head organizer. This is to be attributed to a graded tissue property, called a head activation gradient in much of the hydra literature. In terms of our model, it describes quantitatively the ability of the cells to produce the activator and inhibitor. In our theory of pattern formation, we called this ability the source density. It can be interpreted as a graded competence of the tissue to form the organizing region. Its molecular nature is not yet known. During regeneration, regions with the relatively highest level win the competition and form the organizer.

Why does biology make use of a graded competence in organizer formation? For the generation of the primary body axes, it is most important that only a single organizer can form, despite substantial growth. This is not trivial because it is a property of simple patternforming reactions that new organizing regions are formed whenever a field size becomes larger than the range of the inhibition. Thus, growth would lead to additional organizers and thus to partially fused embryos unless an additional effect is involved, namely a longranging positive feedback of an organizer onto the competence of the tissue to form the organizer (Meinhardt 1993). During growth, cells more distant to the organizer lose more and more of this competence, and therewith the chance to form a second organizer against the inhibition that spreads from the primary organizing region, causing a dramatic increase of the dominance of the primary organizer. A fading competence as assumed in this explanation is a well-known experimental fact. For instance, fragments of a very early chick blastodisc can regenerate a complete embryo even if they do not contain the organizing region (Lutz 1949). This capability for pattern regulation, however, is lost at later stages. Thus, the fading of the competence to form an organizing region is a process of primary importance for suppressing the formation of supernumerary embryos and preventing corresponding malformations. A graded competence can also be established by maternally supplied determinants. This is especially important for embryos starting with large eggs, such as in amphibians to make sure that only a single organizer is formed. This is in contrast to the situation, e.g., in the mouse, in which the embryo is initially so small that multiple organizers can be suppressed although all cells remain competent. In nongrowing systems, such as the sea urchin embryo, a uniform competence is sufficient (Fig. 1). This has the consequence that regeneration can occur with polarity reversal (Hörstadius 1939), in contrast to hydra or planarians.

An organizing region thus exerts two seemingly conflicting effects. On the one hand, it inhibits the formation of other organizing regions. On the other hand, it enhances the competence for organizer formation in the first place. Their separate functions are based on their difference in the time constant. To allow stable patterns and pattern regulation, the inhibitor must have a rapid turnover such that a new organizer can reappear shortly after removal of the original organizer. In contrast, the graded competence needs a long time constant such that within the time scale required for pattern regulation it remains almost unchanged (Fig. 4E,F), providing the bias for the orientation of the regenerating pattern.

\section{UNDER GRADIENT CONTROL: TENTACLE AND FOOT FORMATION}

Hydra tentacles appear in a ring at a certain distance from the primary organizer, the hypostome. The periodic nature of tentacle spacing indicates that their formation is under control of a separate pattern-forming system and does not occur by a simple readout of a gradient that is generated at the tip. Tentacle formation at the correct position can be explained by the assumption that the graded competence generated by and for the primary organizer also represents the gradient of competence for tentacle formation. The primary organizer, however, is the dominant structure. More generally speaking, at long range, the hypostome enables tentacle formation by generating the graded competence. Locally, however, both structures exclude each other. Thus, tentacles 
are formed in the region with the highest competence that is not occupied by the headforming signal and both structures appear necessarily adjacent to each other. This model accounts for the different dynamics of tentacle regeneration, depending on the origin of the fragments along the body column (Fig. 4E,F).

Patterning of foot formation can be explained under the assumption that the foot, i.e., the most anterior structure, appears at the lowest level of head activation gradient and lowers this gradient even further (Fig. 4). Thus, the foot signal contributes to the signaling system that determines the overall polarity of the animal. In a fragment, the foot forms at the largest distance from the head. Head and foot keep the maximum distance within the regenerating fragment, be it big or small, although no direct head/foot inhibition is involved. This is important because a small fragment containing the head does regenerate a new foot in close vicinity of the head. Such regeneration would be impossible if a direct head/foot inhibition would exist.

\section{MULTIPLE HEAD REGENERATION IN PLANARIANS}

Recent observation in planarians suggests a closely related mechanism. In planarians, as in hydra, the canonical Wnt pathway is required to form the posterior organizer, i.e., the tail organizer in planarians (Gurley et al. 2008; Petersen and Reddien 2008; Iglesias et al. 2008; see Adell et al. 2009) or the hypostome in hydra (Hobmayer et al. 2000; see Bode 2009). Down-regulation of the tail organizer in planarians by blocking $\beta$-catenin can lead to the formation of multiple heads. In terms of the model, on blocking $\beta$-catenin, the graded competence would lose its posterior high point. A flat low competence for the posterior organizer would provide the optimal condition for the anterior organizer everywhere in the field. Thus, head formation is no longer restricted to the anterior pole.

Taken together, these observations and their modeling allow several conclusions: (1) The primary organizer keeps nearby cells in a competent state. Cells further away become incompetent for organizer formation, making sure that only a single organizer exists in the system despite the growth. (2) In any tissue fragment, the region pointing toward the original organizer has an advantage in the competition to form the new organizer and will win. Thus, this mechanism accounts for the maintenance of polarity in regenerating fragments. (3) The graded competence (source density, head activation gradient), generated by the primary organizer, acts as a prerequisite to form adjacent structures, whereas a local exclusion makes sure that the two structures do not merge: Structures like head and tentacles appear in a controlled neighborhood. (4) Two organizers can be forced to appear at antipodal positions if each organizer creates local conditions that are nonfavorable for the other-what is good for head formation is bad for foot formation and vice versa. Because there is no direct inhibition between the two organizers, these can emerge close to each other at an early stage - a most important feature to cope with the enormous variation in size, in hydra or planarian development, for example. (5) The use of relative levels for positioning of organizers allows changes of the positional specifications in both directions, toward anterior and toward posterior, in contrast to the distal or posterior transformation discussed above for the interpretation of absolute levels. This is crucial because, in hydra or planarians, both head/foot or head/tail regeneration is possible.

\section{CELL DETERMINATION BOUNDARIES AS ORGANIZING REGIONS FOR SECONDARY EMBRYONIC FIELDS}

A higher organism is much too complex to be generated by a single morphogenetic gradient. Based on his experiments with limb initiation in axolotl, Harrison $(1918,1921)$ proposed that first homogeneous limb fields are formed that subsequently become patterned along the main body axes of the embryo. In an attempt to perform corresponding computer simulations, it turned out that such a scheme would require unrealistic assumptions at the 
Models for the Generation and Interpretation of Gradients

molecular level and that the model was incompatible with Harrison's own data. In a contrasting model, I proposed that such homogeneous limb fields never exist. Instead, new coordinate systems for substructures such as legs and wings in insects and vertebrates are assumed to be generated around the intersections of determination borders (Meinhardt 1982, 1983a,b), e.g., the compartment borders in Drosophila. These borders are formed in a preceding step. The model was able to describe the large body of experiments that were available at those times, including abnormal leg formation after experimental manipulations.
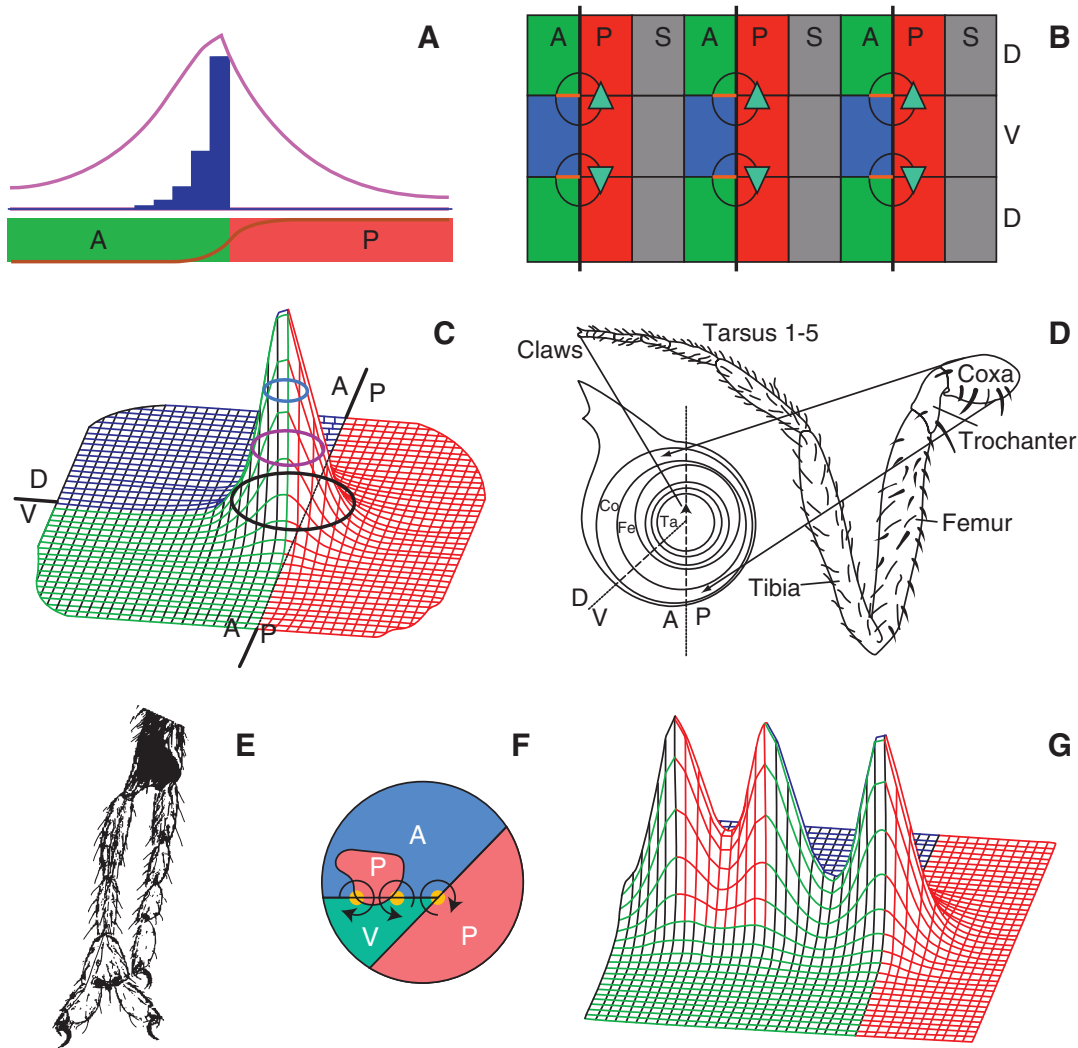

Figure 5. Formation of positional information for substructures at boundaries between differently determined cells (Meinhardt 1982, 1983a,b); the formation of insect legs as an example. $(A)$ in the posterior compartment $(\mathrm{P}$, red) a diffusible cofactor (brown, hedgehog) is produced. It enables morphogen production $(d p p)$ in the anterior compartment (A, green). The region of production (blue) restricted to A-cells that are close to the A/P border. Because of diffusion, a bell-shaped distribution arises (pink). The local concentration is a measure for the distance of a cell from the A/P boundary. (B) Geometry of leg formation. Segmentation was proposed to be based on the repetition of at least three cell states, ... A/PSA/P ... (Meinhardt 1982). Thus, only one A/P border per segment exists. Leg formation requires an intersection of this A/P border with a D/V border. Leg formation occurs in pairs that have opposite handedness (arrow heads). ( $C, D)$ A collaboration of all three compartments $(\mathrm{AD}, \mathrm{AV}$, and $\mathrm{P})$ for a morphogen production leads to a conical morphogen distribution (dll, $E G F)$ that is appropriate to generate the concentric fate map of the insect leg. (E) Heat shock can lead to supernumerary limbs (Girton 1981). The limbs are formed in a plane; the two outer limbs have the normal, the central limb the opposite handedness. $(F, G)$ Model: The heat shock is assumed to cause a flip of some A-cells to a P-specification. The patch of P-cells located on the DV-border in the A-compartment leads to two new intersections along the DV border and thus to two additional limbs. The model-predicted handedness agrees with the observation. 
H. Meinhardt

In the view of the overwhelming evidence that accumulated meanwhile (e.g., Vincent and Lawrence 1994; Martin 1995; Campbell and Tomlinson 1995), the model seems now to be straightforward, if not trivial. At those times, however, this idea was very difficult to publish; accepted only in the fourth journal (Meinhardt 1980). In retrospect, it seems difficult to understand the resistance against this model because it provides a clue why development is so reproducible: The interpretation of a first positional information (Figs. 1 and 2) leads to borders (Fig. 3), which, in turn, give rise to new positional information (Fig. 5) that causes a finer subdivision of the new parts, and so on. Each newly formed structure necessarily has the precise relation to the already established pattern elements. New structures occur in pairs, have the correct handedness on each side of the body, and the correct orientation in relation to the main body axes.

The use of borders as new signaling sources is not restricted to appendage formation. A border between neural tube and ectoderm specification provides, presumably, the signal for neural crest formation, whereas the signal for placodes are formed at the border between neural crest and ectodermal cells (for review, see Schlosser 2008). Borders also play a central role in the further subdivision of the brain (for review, see Joyner 1996; Puelles 2001; Prakash and Wurst 2004; Kiecker and Lumsden 2005).

\section{CONCLUSION}

By using classical observations based on the interference with normal development, we developed molecularly realistic models for basic steps in development. The criterion was that the hypothetical reactions display in computer simulations the same dynamics as found in the experiments. Only a very restricted class of interactions turned out to be conceivable. With the advent of the molecular-genetic approach, it has turned out that the theoretically predicted reactions correspond closely to what is realized, showing that modeling is an appropriate tool to unravel basic principles of development.
The basic reaction types can be summarized as follows:

(i) Primary pattern formation and organizer formation requires local self-enhancement and long-ranging inhibition.

(ii) Permanent activation of a particular gene can be achieved by a positive nonlinear feedback combined with a competition between genes responsible for alternative pathways.

(iii) A dynamic stable neighborhood is enforced if a structure activates on long range its appropriate neighbors but excludes them locally.

(iv) The borders between regions in which different genes are active, and especially the intersection of such borders, can become the new organizing regions to pattern legs, wings, etc., allowing a finer and finer subdivision in an iterative way.

Of course, this list is not complete. Models that describe growth control or pattern formation within individual cells are still in a more rudimentary stage, providing a challenge for future work. Animated simulations for the models discussed above are available at www.eb.tuebingen. mpg.de/meinhardt.

\section{ACKNOWLEDGMENTS}

I wish to express my sincere thanks to Professor Alfred Gierer for a wonderful collaboration over decades.

\section{REFERENCES}

Adell T, Cebrià F, Saló E. 2009. Gradients in planarian regeneration and homeostasis. Cold Spring Harb Perspect Biol 1: a000403.

Barkai N, Shilo B-Z. 2009. Robust generation and decoding of morphogen gradients. Cold Spring Harb Perspect Biol 1: a001990.

Bénazet J-D, Zeller R. 2009. Vertebrate limb development: Moving from classical morphogen gradients to an integrated 4D patterning system. Cold Spring Harb Perspect Biol 1: a001339.

Bode H. 2009. Axial patterning in hydra. Cold Spring Harb Perspect Biol 1: a000463. 
Bode PM, Awad TA, Koizumi O, Nakashima Y, Grimmelikhuijzen CJP, Bode HR. 1988. Development of the two-part pattern during regeneration of the head in hydra. Development 102: 223-235.

Campbell G, Tomlinson A. 1995. Initiation of the proximodistal axis in insect legs. Development 121: 619-628.

Chen Y, Schier AF. 2002. lefty proteins are long-range inhibitors of squint-mediated nodal signaling. Curr Biol 12: 2124-2128.

Christian JL, Moon RT. 1993. Interactions between Xwnt-8 and Spemann organizer signaling pathways generate dorsoventral pattern in the embryonic mesoderm of Xenopus. Genes Dev 7: 13-28.

Dorsky RI, Itoh M, Moon RT, Chitnis A. 2003. Two tcf3 genes cooperate to pattern the zebrafish brain. Development 130: 1937-1947.

Dosch R, Gawantka V, Delius H, Blumenstock C, Niehrs C. 1997. BMP-4 acts as a morphogen in dorsoventral mesoderm patterning in Xenopus. Development 124: $2325-2334$.

Duboc V, Röttinger E, Besnardeau L, Lepage T. 2004. Nodal and BMP2/4 signaling organizes the oral-aboral axis of the sea urchin embryo. Dev Cell 6: 397-410.

Gierer A. 1977a. Biological features and physical concepts of pattern formation exemplified by hydra. Curr Top Dev Biol 11: 17-59.

Gierer A. 1977b. Physical aspects of tissue evagination and biological form. Q Rev Biophys 10: 529-593.

Gierer A, Meinhardt H. 1972. A theory of biological pattern formation. Kybernetik 12: 30-39.

Gierer A, Berking S, Bode H, David CN, Flick K, Hansmann G, Schaller H, Trenkner E. 1972. Regeneration of hydra from reaggregated cells. Nat New Biol 239: 98-101.

Girton JR. 1981. Pattern triplication produced by a celllethal mutation in Drosophila. Dev Biol 84: 164-172.

Gould A, Itasaki N, Krumlauf R. 1998. Initiation of rhombomeric Hoxb4 expression requires induction by somites and a retinoid pathway. Neuron 21: 39-51.

Grapin-Botton A, Bonnin M-A, Sieweke M, Le Douarin NM. 1998. Defined concentrations of a posteriorizing signal are critical for $M a f B /$ Kreisler segmental expression in the hindbrain. Development 125: 1173-1181.

Gurdon JB, Mitchell A, Mahony D. 1995. Direct and continuous assessment by cells of their position in a morphogen gradient. Nature 376: 520-521.

Gurley KA, Rink JC, Sánchez Alvarado A. 2008. $\beta$-Catenin defines head versus tail identity during planarian regeneration and homeostasis. Science 319: 323-327.

Harfe BD, Scherz PJ, Nissim S, Tian F, McMahon AP. 2004. Evidence for an expansion-based temporal Shh gradient in specifying vertebrate digit identities. Cell 118: $517-528$.

Harland R, Gerhart J. 1997. Formation and function of Spemann's organizer. Annu Rev Cell Dev Biol 13: 611-667.

Harrison RG. 1918. Experiments on the development of the fore-limb of Amblystoma, a self-differentiating equipotential system. J Exp Zool 25: 413-461.

Harrison RG. 1921. On relations of symmetry in transplanted limbs. J Exp Zool 32: 1-136.
Hobmayer B, Rentzsch F, Kuhn K, Happel CM, Cramer von Laue C, Snyder P, Rothbächer U, Holstein TW. 2000. WNT signalling molecules act in axis formation in the diploblastic metazoan Hydra. Nature 407: 186-189.

Hörstadius S. 1939. The mechanics of sea-urchin development studied by operative methods. Biol Rev 14: $132-179$.

Iglesias M, Gomez-Skarmeta JL, Saló E, Adell T. 2008. Silencing of smed-ßcatenin 1 generates radial-like hypercephalized planarians. Development 135: 1215-1221.

Joyner AL. 1996. Engrailed, Wnt and Pax genes regulate midbrain hindbrain development. Trends Genet 12: 15-20.

Kiecker C, Lumsden A. 2005. Compartments and their boundaries in vertebrate brain development. Nat Rev Neurosci 6: 553-564.

Kiecker C, Niehrs C. 2001. A morphogen gradient of Wnt/ $\beta$-catenin signalling regulates anteroposterior neural patterning in Xenopus. Development 128: 4189-4201.

Kuziora MA, McGinnis W. 1988. Autoregulation of a Drosophila homeotic selector gene. Cell 55: 477-485.

Lele Z, Nowak M, Hammerschmidt M. 2001. Zebrafish admp is required to restrict the size of the organizer and to promote posterior and ventral development. Dev Dyn 222: 681-687.

Lutz H. 1949. Sur la production experimentale de la polyembryonie et de la monstruosite double ches lez oiseaux. Arch Anat Microsc Morphol Exp 38: 79-144.

Martin GR. 1995. Why thumbs are up. Nature 374: 410-411.

Meinhardt H. 1976. Morphogenesis of lines and nets. Differentiation 6: 117-123.

Meinhardt H. 1978. Space-dependent cell determination under the control of a morphogen gradient. $J$ Theor Biol 74: 307-321.

Meinhardt H. 1980. Cooperation of compartments for the generation of positional information. $Z$ Naturforsch 35c: $1086-1091$.

Meinhardt H. 1982. Models of biological pattern formation. Academic, London. (available at http://www.eb.tuebingen. mpg.de/meinhardt)

Meinhardt H. 1983a. Cell determination boundaries as organizing regions for secondary embryonic fields. Dev Biol 96: 375-385.

Meinhardt H. 1983b. A boundary model for pattern formation in vertebrate limbs. J Embryol Exp Morphol 76: $115-137$.

Meinhardt H. 1993. A model for pattern formation of hypostome, tentacles and foot in Hydra: How to form structures close to each other, how to form them at a distance. Dev Biol 57: 321-333.

Meinhardt H. 2001. Organizer and axes formation as a selforganizing process. Int J Dev Biol 45: 177-188.

Meinhardt H. 2002. The radial-symmetric hydra and the evolution of the bilateral body plan: An old body became a young brain. Bioessays 24: 185-191.

Meinhardt H. 2004. Different strategies for midline formation in bilaterians. Nat Rev Neurosci 5: 502-510.

Meinhardt H. 2006. Primary body axes of vertebrates: Generation of a near-Cartesian coordinate system and 


\section{H. Meinhardt}

the role of Spemann-type organizer. Dev Dyn 235: 2907-2919.

Meinhardt H. 2008. Models of biological pattern formation: From elementary steps to the organization of embryonic axes. Curr Top Dev Biol 81: 1-63.

Moos M, Wang SW, Krinks M. 1995. Anti-dorsalizing morphogenetic protein is a novel TGF- $\beta$ homolog expressed in the Spemann organizer. Development 121: 4293-4301.

Nakamura T, Mine N, Nakaguchi E, Mochizuki A, Yamamoto M, Yashiro K, Meno C, Hamada M. 2006. Generation of robust left-right asymmetry in the mouse embryo requires a self-enhancement and lateralinhibition system. Dev Cell 11: 495-504.

Nieuwkoop PD. 1952. Activation and organization of the central nervous system in amphibians. III. Synthesis of a new working hypothesis. J Exp Zool 120: 83-108.

Nieuwkoop PD. 1992. The formation of the mesoderm in urodelean amphibians. VI. The self-organizing capacity of the induced meso-endoderm. Roux's Arch Dev Biol 201: 18-29.

Nordström U, Jessell TM, Edlund T. 2002. Progressive induction of caudal neural character by graded Wnt signaling. Nat Neurosci 5: 525-532.

Nunes da Fonseca R, von Levetzow C, Kalscheuer P, Basal A, van der Zee M, Roth S. 2008. Self-regulatory circuits in dorsoventral axis formation of the short-germ beetle Tribolium castaneum. Dev Cell 14: 605-615.

Petersen CP, Reddien PW. 2008. Smed+Bcatenin-1 is required for anteroposterior blastema polarity in planarian regeneration. Science 319: 327-330.

Plouhinec JL, De Robertis EM. 2009. Systems biology of the self-regulating morphogenetic gradient of the Xenopus gastrula. Cold Spring Harb Perspect Biol 1: a001701.

Prakash N, Wurst W. 2004. Specification of midbrain territory. Cell Tissue Res 318: 5-14.

Puelles L. 2001. Brain segmentation and forebrain development in amniotes. Brain Res Bull 55: 695-710.
Regulski M, Dessain S, McGinnis N, McGinnis W. 1991. High-affinity binding-sites for the deformed protein are required for the function of an autoregulatory enhancer of the Deformed gene. Genes Dev 5: 278-286.

Reversade B, De Robertis E. 2005. Regulation of ADMP and BMP2/4/7 at opposite embryonic poles generates a selfregulating morphogenetic field. Cell 123: 1147-1160.

Ringrose L, Paro R. 2007. Polycomb/trithorax response elements and epigenetic memory of cell identity. Development 134: 223-232.

Saùde L, Woolley K, Martin P, Driever W, Stemple DL. 2004. Axis-inducing activities and cell fates of the zebrafish organizer. Development 127: 3407-3417.

Schlosser G. 2008. Do vertebrate neural crest and cranial placodes have a common evolutionary origin? Bioessays 30: $659-672$.

Schwartz YB, Pirrotta V. 2007. Polycomb silencing mechanisms and the management of genomic programmes. Nat Rev Genet 8: 9-22.

Starz-Gaiano M, Melani M, Wang X, Meinhardt H, Montell DJ. 2008. Feedback Inhibition of JAK/STAT signaling by apontic is required to limit an invasive cell population. Dev Cell 14: 726-738.

Turing A. 1952. The chemical basis of morphogenesis. Philos Trans R Soc Lond B Biol Sci 237: 37-72.

Vincent JP, Lawrence PA. 1994. It takes three to distalize. Nature 372: 132-133.

Willot V, Mathieu J, Lu Y, Schmid B, Sidi S, Yan YL, Postlethwait JH, Mullins M, Rosa F, Peyrieras N. 2002. Cooperative action of ADMP- and BMP-mediated pathways in regulating cell fates in the zebrafish gastrula. Dev Biol 241: 59-78.

Yuan S, Schoenwolf GC. 1998. De-novo induction of the organizer and formation of the primitive streak in an experimental-model of notochord reconstitution in avian embryos. Development 125: 201-213. 


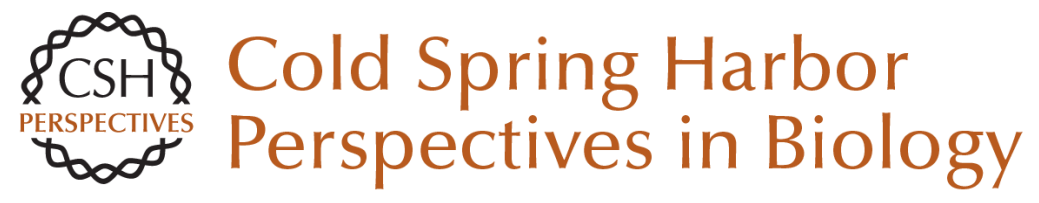

\section{Models for the Generation and Interpretation of Gradients}

Hans Meinhardt

Cold Spring Harb Perspect Biol 2009; doi: 10.1101/cshperspect.a001362 originally published online July 15, 2009

\section{Subject Collection Generation and Interpretation of Morphogen Gradients}

Regulation of Organ Growth by Morphogen Gradients

Gerald Schwank and Konrad Basler

Signaling Gradients during Paraxial Mesoderm

Development Alexander Aulehla and Olivier Pourquié

Morphogen Gradient Formation

Ortrud Wartlick, Anna Kicheva and Marcos

González-Gaitán

Nodal Morphogens

Alexander F. Schier

Gradients and the Specification of Planar Polarity

in the Insect Cuticle

David Strutt

Vertebrate Limb Development: Moving from

Classical Morphogen Gradients to an Integrated

4-Dimensional Patterning System Jean-Denis Bénazet and Rolf Zeller

Establishing and Interpreting Graded Sonic

Hedgehog Signaling during Vertebrate Neural

Tube Patterning: The Role of Negative Feedback Vanessa Ribes and James Briscoe

Systems Biology of the Self-regulating

Morphogenetic Gradient of the Xenopus Gastrula Jean-Louis Plouhinec and E. M. De Robertis
Gradients in Planarian Regeneration and Homeostasis

Teresa Adell, Francesc Cebrià and Emili Saló

Shaping Morphogen Gradients by Proteoglycans Dong Yan and Xinhua Lin

Forming Patterns in Development without Morphogen Gradients: Scattered Differentiation and Sorting Out

Robert R. Kay and Christopher R.L. Thompson

Robust Generation and Decoding of Morphogen Gradients

Naama Barkai and Ben-Zion Shilo

Models for the Generation and Interpretation of

Gradients

Hans Meinhardt

Graded Dorsal and Differential Gene Regulation in the Drosophila Embryo

Gregory T. Reeves and Angelike Stathopoulos

Chemical Gradients and Chemotropism in Yeast Robert A. Arkowitz

Gradients in the Brain: The Control of the Development of Form and Function in the Cerebral Cortex

Stephen N. Sansom and Frederick J. Livesey

For additional articles in this collection, see http://cshperspectives.cshlp.org/cgi/collection/

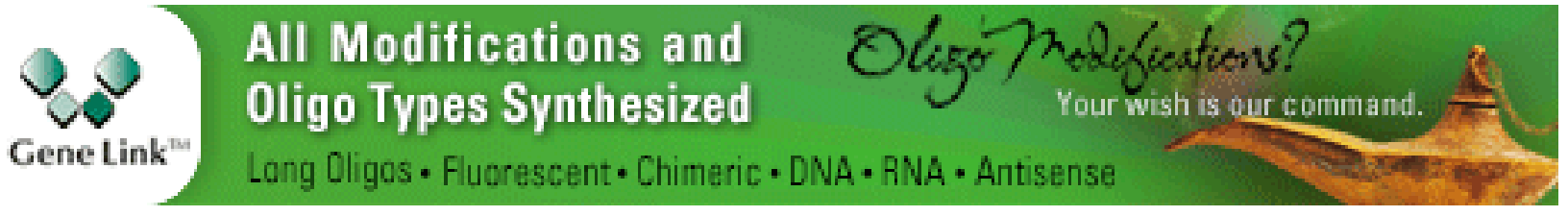


For additional articles in this collection, see http://cshperspectives.cshlp.org/cgi/collection/

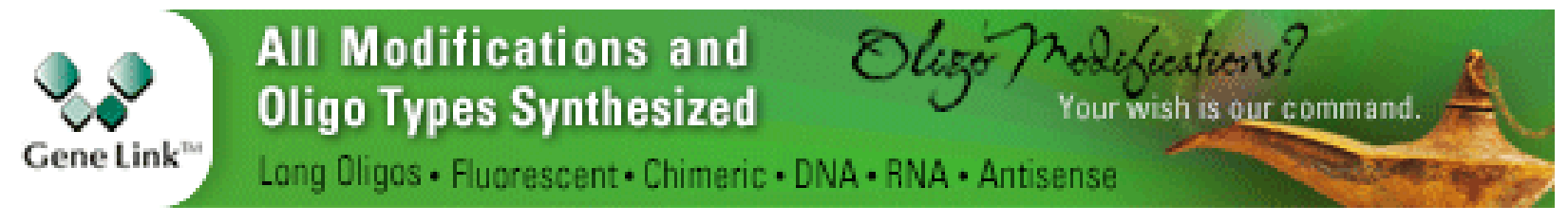

Copyright @ 2009 Cold Spring Harbor Laboratory Press; all rights reserved 